\title{
A Narrative Review of Norovirus Gastroenteritis: More Global Attention Is Needed
}

\author{
Cheng $\mathrm{Xu}^{1}$, Jianguang $\mathrm{Fu}^{2}$, Yefei Zhu ${ }^{{ }^{*}}$ \\ ${ }^{1}$ Laboratory Medical Center, The Second Affiliated Hospital of Nanjing Medical University, Nanjing, China \\ ${ }^{2}$ Key Lab of Enteric Pathogenic Microbiology, Ministry of Health, Jiangsu Provincial Center for Disease Control and Prevention, \\ Nanjing, China
}

Corresponding Author: Yefei Zhu, M.D., Professor, Laboratory Medical Center, The Second Affiliated Hospital of Nanjing Medical University, 121 Jiangjiayuan, Nanjing, 210011, Jiangsu Province, P.R. China. Tel: +86-18951727659; Email: zhuyf@njmu.edu.cn

Received November 7, 2016; Accepted November 25, 2016; Online Published December 5, 2016

\begin{abstract}
Noroviruses (NoVs), an important pathogenic agent of foodborne illness, lead to acute and chronic gastroenteritis in humans of all ages and to travelers' diarrhea. Many genotypes exist in nature; Gll.4 is widely known as the most predominant. Outbreaks mostly occur in semi-closed settings. Although the disease is self-limited to person, many cases have resulted in death, which has raised more concerns. However, a lack of microbial culture techniques limits research and thus knowledge about these viruses. To date, there are no specific antiviral drugs that fight NoVs. Rehydration is the ideal approach at present for severe cases. The only way to prevent infection is to improve personal hygiene. So many variants and ambiguous evolution mechanisms make research for a vaccine much more difficult. In recent years, several vaccine candidates entered pre-clinical development. This review concentrates on summarizing the aspects of NoV structure, culture, genetic evolution, and the vaccine.
\end{abstract}

Keywords: Norovirus, Epidemiology, Diagnosis, Vaccine

Citation: Xu C, Fu J, Zhu Y. A narrative review of norovirus gastroenteritis: more global attention is needed. Int J Travel Med Glob Health 2016;4(4):101-106. doi:10.21859/ijtmgh-040402.

\section{Introduction}

Acute gastroenteritis (diarrhea and vomiting) leads to 1.45 million deaths worldwide every year and is considered one of the greatest burdens of all infectious diseases, second only to respiratory infections. ${ }^{1}$ Acute gastroenteritis can be caused by bacteria or viruses; the main viral pathogens of acute gastroenteritis are rotaviruses and Noroviruses (NoVs). Since the implementation of rotavirus vaccines in many countries, NoVs have replaced rotaviruses as the main etiologic agent of viral epidemic gastroenteritis. ${ }^{2}$ They impose staggering economic costs and social burdens. ${ }^{3}$ To date, there is no sufficient therapy or commercial vaccine for NoVs. ${ }^{4}$

\section{Viral Particle Structure and Function}

$\mathrm{NoV}$ was first discovered using electron microscopy (EM). It is a small, round (27-38 $\mathrm{nm}$ in diameter), non-enveloped, icosahedral virus belonging to the family Caliciviradae under the genus Norovirus. The NoV genome consists of a single positive-sense RNA which is approximately 7.5-7.7 $\mathrm{kb}$ and encodes three open reading frames (ORFs) ${ }^{5}$ with the exception of the murine norovirus (MNV). MNV has a fourth alternative ORF. ${ }^{6}$ The first ORF (ORF1) encodes a large nonstructural polyprotein which is post-translationally cleaved by a virus-encoded protease (Pro; NS6) into six or seven mature nonstructural proteins involved in viral replication ${ }^{7}$ from the $\mathrm{N}$ to the $\mathrm{C}$ terminus including $\mathrm{N}$-terminal protein (designated $\mathrm{p} 48$ for Norwalk virus; NS12), NTPase (p41; NS3), 3A-like protein (designated p22 for Norwalk virus; NS4), a viral genome-linked protein (VPg; NS5), viral protease (3Clpro; NS6), ${ }^{8}$ and RNA-dependent RNA polymerase (RdRp; NS7). ${ }^{9}$ The ORF2 and ORF3 encode the major capsid protein $\mathrm{VP} 1$ and the minor structure protein VP2, respectively. The icosahedral T3 recombinant NoV is formed by assembly of the 90 dimers of the VP1 capsid protein. VP1 is $1.8 \mathrm{~kb}$ in length and organized into two principal domains, the shell $(\mathrm{S})$ and protrusion $(\mathrm{P})$ domains. The $\mathrm{S}$ domain is highly conserved, which is related to the formation of the NoV's structure. The P domain can form virus-like particles, even with 20 aa missing in the $\mathrm{N}$ terminal, and still have no effect on the ability to function as a receptor. ${ }^{10}$ The P domain is further subdivided into the P1 and P2 subdomains. The moderately flexible P1 sub-domain acts as a hinge to combine $\mathrm{S}$ and $\mathrm{P} 2$ sub-domains. The $\mathrm{P} 2$ sub-domain is located on the most distal surface and is highly variable. It

Copyright $\odot 2016$ The International Journal of Travel Medicine and Global Health. This is an open-access article distributed under the terms of the Creative Commons Attribution License (http://creativecommons.org/licenses/by/4.0), which permits unrestricted use, distribution, and reproduction in any medium, provided the original work is properly cited. 
includes the sequences of binding carbohydrates and blood group antigen, combinations associated with the host cell. The P2 sub-domain is closely related to the popularity of the mutant, which suggests that this area should contain specific antigenic determinants, receptor binding sites, and neutralize antibody binding sites. Bertolotti-Ciarlet et al suggests that VP2 is associated with the upregulation and stabilization of VP1 in the virus. ${ }^{11}$

\section{Classification and Nomenclature}

The diversity of NoV is extensive, and the specific classification is significantly important as different virus types can differ in many aspects, such as virulence, environmental stability, and histotropism. However, direct serotyping by neutralization assays is impossible because of the lack of a cell culture system. Researchers proposed a criteria to classify NoVs below the genus level based on the amino acid sequences of the VP1 major capsid protein and $\mathrm{RdRp}^{12}$ with the widespread use of reverse transcription-polymerase chain reaction (RT-PCR) and genetic sequencing. Now, NoVs can be correctly classified by whole genome sequencing as more antigenic drifts and recombination events occur. ${ }^{13}$ Accordingly, at least 40 genotypes are segregated into seven genogroups (GI-GVII), ${ }^{14}$ including 9 genotypes in the GI genogroup, 22 in GII, 3 in GIII, 2 in GIV, 2 in GV, 2 in GVI, and 1 in GVII. Three of the genogroups (GI, GII, and GIV) are primarily found in human infections, except for GII.11, GII.18, and GII.19 in pigs. ${ }^{15,16}$ GII.4 has the most variants and is responsible for the most outbreaks and sporadic diseases (>95\% of NoV infections). GIII and GV infect bovine and mice, respectively. The new genogroup GVII was recently proposed; it was initially detected in dogs, ${ }^{17}$ which raised great concerns over possible inter-species transmission.

As there is so much diversity, it can be assumed that NoVs evolve faster than any other RNA virus. More research is needed to add evidence for dynamic evolution.

Traditional nomenclature used to be a form of genogroupgenotype combination, for example, GI.1 or GI/1, with the roman number standing for genogroup and the Arabic number representing the genotype. The new style of nomenclature is as specific as possible: genotype/host/country/isolation year/ partial ORF1-ORF2-strain name and index year/isolate city name (usually with an isolate number). ${ }^{18}$

\section{Culture}

Since the discovery of NoVs in the 1970s, most attempts to establish an efficient cell culture system coupled with an animal model have obtained negative results. Thus, studies of survival, replication, gene expression, or immunology cannot be conducted. However, the three major methods for $\mathrm{NoV}$ model development, constructing in vitro cell culture system models with potential human and animal cell lines, infecting animal hosts with NoVs, and using animal caliciviruses as surrogates in their natural hosts, provide much encouragement.

\section{Cell Culture System}

Considering the enteric nature of NoVs, researchers try to mimic the intestinal environment, but no human or animal cell line supports the significant replication of NoVs. MNV has been found to replicate in dendritic cells and macrophages in murine hosts. ${ }^{19}$ Based on this observation, some researchers have utilized NoVs transfecting such human cell types, but no increase in NoV RNA was detected in any cell. ${ }^{20}$ A single group of researchers successfully investigated NoV replication in three-dimensional cultures using human intestinal epithelial cell lines. ${ }^{21}$ Mary K. Estes along with her research team successfully cultured $\mathrm{HuNoV}$ strains in stem cell-derived human intestinal enteroids (HIEs). Moreover, the team also confirmed that bile is necessary for the replication of some NoV strains. ${ }^{22}$ This finding is extremely exciting and must not only be the impetus of basic pathogenesis research, but also provide a platform for vaccine and anti-viral drug development.

\section{Animal Models}

Animal models, namely chimpanzees, gnotobiotic (Gn) pigs, and $\mathrm{Gn}$ calves, are being used to increase what is understood about NoVs. ${ }^{23-25}$ Chimpanzees showed subclinical infection but had a similar duration of virus shedding and level of replication as found in humans. Pigs and calves exhibited the same symptoms as humans, partially due to their similar gut structure and physiology. However, the high cost of Gn animals and the NoV strain-specific infection limited their feasibility.

\section{Surrogated Virus}

To date, most of the data on NoV comes from the study of surrogates, such as MNV, feline calicivirus (FCV), and Tulane virus (TV). ${ }^{26-28} \mathrm{MNV}$ has become the most frequently used NoV surrogate as it is the only NoV with a cell culture system and small animal model. TV also has broad uses as a surrogate since it has similar genetic identity to NoVs and recognizes histoblood group antigens (HBGA). However, whether surrogates accurately represent human NoVs is frequently questioned. ${ }^{29}$ Continuous development of new surrogate models providing capabilities for both in vitro and in vivo studies is still needed to overcome the limitations of current systems.

\section{Epidemiology and Clinical Features}

Since the first NoV infection was recorded in Norwalk, the United States in 1968, ${ }^{30}$ NoV-related gastroenteritis has been more frequently reported, accounting for at least $90 \%$ of nonbacterial acute gastroenteritis and more than $50 \%$ of all-cause outbreaks worldwide. ${ }^{31}$ Each year in the United States, NoV causes on average 19-21 million cases of acute gastroenteritis and leads to 1.7-1.9 million outpatient visits and 400000 emergency department visits, primarily for young children. Worldwide, about one out of every five cases of acute gastroenteritis is caused by NoV, and these cases most often occur in developing countries. ${ }^{32}$ Furthermore, NoV has shared a common etiology with travelers' diarrhea in recent years. ${ }^{33}$ Overall, GII.4 is the most predominant genotype reported in most outbreaks, circulating with an emerging variant every 2-3 years that appears to escape human herd immunity to previous strains. ${ }^{34,35}$ Such outbreaks involve the general population across all ages, particularly those at the 
extremes of age and some who are immunocompromised or have received organ transplants. ${ }^{36}$ The seasonality of NoV outbreaks is obvious, with infection peaking during the dry winter months. Outbreaks typically occur in semi-closed settings where people are in close contact with each other, such as hospital wards, healthcare centers, cruise ships, schools, and hotels. ${ }^{37-40}$ There is even a report of NoV transmission during an international flight, pointing out that the risk of $\mathrm{NoV}$ infection on a commercial flight is likely greater on longer flights. ${ }^{41}$ NoVs are mainly transmitted through the fecal-oral route by contact with infected individuals or contaminated food/water, but they may also occur through exposure to airborne particles from explosive vomitus or stool. ${ }^{42}$ Several factors make NoVs highly contagious: (1) NoV particles are environmentally stable and much more resistant to heat, $\mathrm{pH}$, and disinfection than bacteria; (2) low-dose particles (usually 18 to 2800 viral particles) can be sufficient to cause infection ${ }^{43}$; (3) virus shedding precedes the onset of symptoms and continues for 2 weeks after return to baseline health; and (4) repeated infections can occur due to lack of cross-protection against the diverse $\mathrm{NoV}$ strains and inadequate long-term immunity. ${ }^{44}$

The incubation period is estimated as 1.2 days (95\% CI, 1.1 to 2.2 days). ${ }^{45}$ The disease is often characterized by vomiting, non-bloody diarrhea, abdominal cramps, lowgrade fever, mild, self-limited, and lasting 1-3 days. Besides the self-limited cases, it is more important for the severe cases. NoVs contribute to about 570-800 deaths per year on average, mostly among young children and the elderly. Asymptomatic infections also exist and are estimated to occur in approximately one-third of infected persons. They have no specific symptoms, but active virus shedding can occur with both infected and asymptomatic patients and peak on the fourth day following exposure. ${ }^{46}$

\section{Evolution Mechanism}

Many studies of outbreaks have identified NoVs of the GII genogroup as the most common strains worldwide, which suggests that NoV GII strains have rapidly evolved with high levels of genetic divergence and adaptation to humans. Two mechanisms of variation, mutation and homologous recombination, are recognized contributors to the evolution of NoVs.

Under the herd immune pressure, variation of the P2 subdomain results in differential HBGA binding patterns which lead to changes in host susceptibility. ${ }^{47}$ Five epitopes (A-E) within the capsid P2 domain were observed as the most common variation sites among different GII.4 variants, especially epitope A. ${ }^{34}$ Eden et al conducted a study which confirmed that antigenic variation was an important factor contributing to the emergence of novel NoVs, and four sites within the P2 domain $(294,368,373$, and 376) were identified with novel, variant specific mutations. Among them, two sites (294 and 368) were located in epitope A, one (376) was in epitope C, and another site (373) was not associated with any previously defined epitope. ${ }^{48}$ The slow accumulation of mutations along the P2 subdomain eventually resulted in a novel GII.4 strain with the potential to escape herd immunity, which altered antigenicity and HBGA binding properties. ${ }^{49}$
This observation supports the notion of NoV evolution that has been driven by population immunity.

Successful RNA viruses have been shown to evade host immunity through several modes, including the effect of replication fidelity. The lack of related proofreading and repair mechanisms for RNA replication and transcription makes the RNA virus mutation rate significantly higher than all extant organisms with an estimated mutation rate in the range of $10^{-3}$ to $10^{-5} .^{50}$ The substantial production of mutants supports the emergence of variants with potentially useful phenotypes in the face of environmental change. However, with the increase of mutations, the virus fitness would decrease and finally deracinate if it eventually passes the error threshold. ${ }^{51}$ Two prevalent genotypes, GII.4 and recombinant GII.12/GII.3, are excellent examples that maintain the balance between mutation and fidelity.

The ability of RNA polymerases to switch templates facilitates the development of recombinant RNA, which is another major driving force of viral evolution and a powerful mechanism for creating large changes in the viral genome. ${ }^{52}$ Recombination in NoVs may play a significant role in limiting humoral cytotoxic T-lymphocyte immune responses. There exist two types of recombination, inter-genotype and intra-genotype. Most recombination in NoV occurs at the ORF1-ORF2 overlap, ${ }^{53}$ which is also the transcription start site for viral subgenomic RNAs. ${ }^{54}$ Intragenic recombination also has been reported to locate at the ORF2-ORF3 junction. ${ }^{55}$ Recombination within the capsid ORFs transforms the orientation of the capsid domains and therefore prevents neutralization by pre-existing antibodies so that the NoV escapes human immunity. There are many recombinant NoVs, including GIIPb/GII.3, GII.Pb/GII.13, GII.Pg/GII.12, GII. P4/GII.10, GII.P6/GII.14, and so on, and nearly all of them are non-GII.4 genotypes. To date, 11 recombination variants have been reported. They mainly result in epidemics among children rather than widespread gastroenteritis pandemics. ${ }^{56}$ Mutation and recombination drives the continual evolution of NoVs, which means that drugs and vaccines against NoV will be in the early phase period for a long time.

\section{Diagnosis \\ Clinical Test}

NoV infections can be detected on the basis of the following clinical and epidemiological features which are different from other causes of enteric disease ${ }^{57}:(1)$ a mean incubation period of 24-48 hours; (2) vomiting ( $>50 \%$ of patients); (3) a short duration of 12-60 hours; (4) Exclusion of common bacteria, parasites, and other pathogens. It is confirmed that this set of criteria is highly specific (99\%) and moderately sensitive $(68 \%) .^{58}$ This information is very useful for testing NoVs in the community and in hospitals when there is no laboratory diagnosis available.

\section{Electron Microscopy}

EM was the first method to identify NoV according to their morphology. ${ }^{59}$ EM can provide a rapid test (15 minutes) and is able to detect a range of viral pathogens. However, it is no longer an adequate detection method because of its expensive facilities and low sensitivity. In addition, the detection 
results are directly related to the skills and experience of the operator; therefore, it is not widely available for large-scale epidemiological investigation.

\section{Immunological Tests}

Immunological methods include radioimmunoassay (RIA), enzyme-linked immunosorbent assay (ELISA), and immunochromatography (ICG). The sensitivity of RIA can be increased 10-20-fold that of EM. RIA can also detect serum antibody rises and offer more valuable information for epidemiology. However, it is time consuming (6D) and requires a radioactive isotope, which limits its extensive use in laboratories. ELISA is a rapid and economic method, and its scope of application is relatively narrow because of the high specificity between strains. Now, it is mainly used to screen large numbers of fecal samples for NoVs with relative ease. ICG does not require laboratory equipment and offers the advantage of rapid (20 minutes) individual sample testing. Recent evolving technologies have given the ICG test an analytical sensitivity ranging between $23 \%$ and $59 \%$ with a specificity ranging $75 \%-100 \% .{ }^{60}$ The sensitivity rates of ICG tests by different manufacturers are significantly different, which may be related to the difference in viral loads among samples. ${ }^{61}$ To sum up, the use of immunoassays can simplify the testing and diagnosis of NoV infection, but the obviously decreased sensitivity when compared to molecular testing limits its widespread use.

\section{Molecular Tests}

Once the sequence of NoV was known, RT-PCR was introduced in the mid-1990s. It was a reference method with high analytical sensitivity and specificity. The conventional RT-PCR amplicons can be sequenced and used for typing, but its results must be confirmed by sequencing or hybridization, and the process is time consuming (5-6 hours). With the development of diagnostic technologies, real-time RT-PCR has replaced RT-PCR and is now the gold standard for the clinical detection of NoVs. This method is more sensitive, faster ( 3 hours), and has a high throughput, providing both confirmation and quantitation in a single assay. Both ELISA and molecular tests are challenging because of the diversity of strains and the antigenic drift with time.

The need for rapid diagnosis should be balanced against the accuracy of reporting, especially because of the infection control implications. Therefore, each laboratory needs to select a testing format that is best suited to its needs and feasibilities.

\section{Vaccine}

There is no specific antiviral therapy at present. Rehydration with fluids and electrolytes is an ideal approach for clinical severe cases. Improving hand hygiene is the most important, simplest, and least expensive method of preventing $\mathrm{NoV}$ infections. The viruses are resistant to routine cleansing and routine alcohol-base hand hygiene solutions.

A potential vaccine is needed to control the disease. Previous investigators were devoted to utilizing bioengineering technologies to produce recombinant $\mathrm{NoV}$ antigens while lacking a suitable cell culture system or animal model, including mainly NoV-like particle vaccines (VLPs) and $\mathrm{P}$ particles.

VLP vaccines are low-cost and can be produced in many expression systems, including yeast, ${ }^{62}$ baculovirus-infected insect cells, ${ }^{63}$ and transgenic plants. ${ }^{64,65}$ VLPs are selfassembled by NoV VP1 capsid sequences, and P particles are expressed by the P domain of VP1 capsid protein. Both are morphologically similar to the natural virus and have similar properties. ${ }^{63,66}$ In preclinical studies, these vaccine candidates were administered to mice and $\mathrm{Gn}$ pigs, respectively. The results confirmed that they are highly immunogenic and can produce antibodies. ${ }^{67,68}$ Other studies have shown a dose-dependent response of the VLP vaccine; when the vaccine quantity was increased, the neutralizing antibody quantity increased significantly on the second day after the second vaccination. ${ }^{69}$ Clinical trials to assess the safety, immunogenicity, and efficacy of the VLP vaccine in volunteers are ongoing. ${ }^{70}$ Some adverse reactions have been observed. In the vaccine groups, three cases of mild cramps and one case of nausea were reported. Those who received the vaccine through the intranasal route more commonly reported mild nasal stuffiness and sneezing. Volunteers in the vaccine and placebo groups both had the systemic symptoms of headache and malaise. The first generation VLP vaccines have reached phase II clinical trials and promise a positive outcome. ${ }^{4}$

However, with the report of a successful cell culture system, the focus should be placed on traditional strategies of live attenuate and inactivated vaccine.

Many challenges still hinder the development of a vaccine. Considering the genetic diversity and antigenic drift of NoVs, it is necessary to seek polyvalent vaccines with broad protection. Consequently, real-time monitoring the epidemiology of NoVs is needed for future vaccine improvement. Furthermore, NoV infection is species specific; hence, the data from animal models maybe limited and inaccurate. Overall, no vaccine is available, but vaccine studies are ongoing.

\section{Conclusion}

What has been learned about NoVs is encouraging. Areas in NoV research that await investigation include (1) The 3D structure of ssRNA and the specific function of the related protein in the replication progress; (2) Details of the NoV viroplasm interaction with HBGA in intestinal mucosa; (3) The impact of NoV infection on the long-term health of the gut; (4) The mechanisms controlling mutation/reassortment in the replication progress; (5) The factors determining NoV pathogenicity and virulence; (6) The significance of the different immune response types in establishing protection against NoVs; (7) The factors determining the spread of NoV; (8) The development of alternative (non-live attenuated) NoV vaccine candidates and anti-viral drugs.

\section{Authors' Contributions}

YZh conceived of this work and revised the manuscript. ChX and JF wrote the manuscript. All authors read and approved the final manuscript.

\section{Conflict of Interest Disclosures}

There is no conflict of interest. 
Review Highlights

\section{What Is Already Known?}

NoVs are important, acute pathogenic agents. The aspects of NoV structure, culture, genetic evolution, and vaccine are summarized .More typical references and epidemiological data on NoVs is added to emphasize the disease more than before. Through long-term monitoring, we have known well about the clinical symptoms, epidemiological characteristics and evolution mechanism. Besides, some other aspects of NoVs (such as culture) come from the MNV or other surrogates. Real-time monitoring is necessary because of genetic diversity and the antigenic drift.

\section{What This Study Adds?}

This study clearly adds some new findings (e.g., the new GII.4 variant, new epidemiological date, new methods of culture, and so on) resulting from the authors' own work and that of other researchers. Some ideas for further research are also suggested.

\section{Ethical Approval}

There is no ethical aspect to the article.

\section{Acknowledgments}

This work was supported by the Jiangsu Province Science \& Technology Project of Clinical Medicine (BL2014081) and the Jiangsu Province Health Development Project with Science \& Education (RC2011085).

\section{References}

1. Ahmed SM, Hall AJ, Robinson AE, et al. Global prevalence of norovirus in cases of gastroenteritis: a systematic review and metaanalysis. Lancet Infect Dis. 2014;14(8):725-730. doi:10.1016/ S1473-3099(14)70767-4.

2. Fischer Walker CL, Perin J, Aryee MJ, Boschi-Pinto C, Black RE. Diarrhea incidence in low- and middle-income countries in 1990 and 2010: a systematic review. BMC Public Health. 2012;12:220. doi:10.1186/1471-2458-12-220.

3. White PA. Evolution of norovirus. Clin Microbiol Infect. 2014;20(8):741-745. doi:10.1111/1469-0691.12746.

4. Tan $M$, Jiang $X$. Vaccine against norovirus. Hum Vaccin Immunother. 2014;10(6):1449-1456. doi:10.4161/hv.28626.

5. Alhatlani B, Vashist S, Goodfellow I. Functions of the $5^{\prime}$ and $3^{\prime}$ ends of calicivirus genomes. Virus Res. 2015;206:134-143. doi:10.1016/j.virusres.2015.02.002.

6. McFadden N, Bailey D, Carrara G, et al. Norovirus regulation of the innate immune response and apoptosis occurs via the product of the alternative open reading frame 4. PLoS Pathog. 2011;7(12):e1002413. doi:10.1371/journal.ppat.1002413.

7. May J, Viswanathan P, Ng KK, Medvedev A, Korba B. The p4-p2' amino acids surrounding human norovirus polyprotein cleavage sites define the core sequence regulating self-processing order. J Virol. 2014;88(18):10738-10747. doi:10.1128/JVI.01357-14.

8. Hussey RJ, Coates L, Gill RS, et al. A structural study of norovirus 3C protease specificity: binding of a designed active site-directed peptide inhibitor. Biochemistry. 2011;50(2):240-249. doi:10.1021/ bi1008497.

9. Ayukekbong JA, Mesumbe HN, Oyero OG, Lindh M, Bergstrom T. Role of noroviruses as aetiological agents of diarrhoea in developing countries. J Gen Virol. 2015;96(8):1983-1999. doi:10.1099/vir.0.000194

10. Bertolotti-Ciarlet A, White LJ, Chen R, Prasad BV, Estes MK.
Structural requirements for the assembly of Norwalk virus-like particles. J Virol. 2002;76(8):4044-4055.

11. Bertolotti-Ciarlet A, Crawford SE, Hutson AM, Estes MK. The 3' end of Norwalk virus mRNA contains determinants that regulate the expression and stability of the viral capsid protein VP1: a novel function for the VP2 protein. J Virol. 2003;77(21):11603-11615.

12. Zheng DP, Ando T, Fankhauser RL, Beard RS, Glass RI, Monroe SS. Norovirus classification and proposed strain nomenclature. Virology. 2006;346(2):312-323. doi:10.1016/j.virol.2005.11.015.

13. Hernandez JD, Silva LD, Sousa ECJ, et al. Analysis of uncommon norovirus recombinants from Manaus, Amazon region, Brazil: GIl.P22/GII.5, GIl.P7/GII.6 and GIl.Pg/GIl.1. Infect Genet Evol. 2016;39:365-371. doi:10.1016/j.meegid.2016.02.007.

14. Caddy S, Breiman A, le Pendu J, Goodfellow I. Genogroup IV and $\mathrm{VI}$ canine noroviruses interact with histo-blood group antigens. J Virol. 2014;88(18):10377-10391. doi:10.1128/JVI.01008-14.

15. Wang QH, Costantini V, Saif LJ. Porcine enteric caliciviruses: genetic and antigenic relatedness to human caliciviruses, diagnosis and epidemiology. Vaccine. 2007;25(30):5453-5466. doi:10.1016/j.vaccine.2006.12.032.

16. Shen Q, Zhang W, Yang S, Cui L, Hua X. Complete genome sequence of a new-genotype porcine norovirus isolated from piglets with diarrhea. J Virol. 2012;86(12):7015-7016. doi:10.1128/JVI.00757-12.

17. Martella V, Lorusso E, Decaro N, et al. Detection and molecular characterization of a canine norovirus. Emerg Infect Dis. 2008;14(8):1306-1308. doi:10.3201/eid1408.080062.

18. Moore MD, Goulter RM, Jaykus LA. Human norovirus as a foodborne pathogen: challenges and developments. Annu Rev Food Sci Technol. 2015;6:411-433. doi:10.1146/annurevfood-022814-015643.

19. Wobus CE, Karst SM, Thackray LB, et al. Replication of Norovirus in cell culture reveals a tropism for dendritic cells and macrophages. PLoS Biol. 2004;2(12):e432. doi:10.1371/journal.pbio.0020432.

20. Lay MK, Atmar RL, Guix S, et al. Norwalk virus does not replicate in human macrophages or dendritic cells derived from the peripheral blood of susceptible humans. Virology. 2010;406(1):111. doi:10.1016/j.virol.2010.07.001.

21. Takanashi S, Saif LJ, Hughes JH, et al. Failure of propagation of human norovirus in intestinal epithelial cells with microvilli grown in three-dimensional cultures. Arch Virol. 2014;159(2):257-266. doi:10.1007/s00705-013-1806-4.

22. Ettayebi K, Crawford SE, Murakami K, et al. Replication of human noroviruses in stem cell-derived human enteroids. Science. 2016. doi:10.1126/science.aaf5211.

23. Bok K, Parra Gl, Mitra T, et al. Chimpanzees as an animal model for human norovirus infection and vaccine development. Proc Natl Acad Sci U S A. 2011;108(1):325-330. doi:10.1073/ pnas.1014577107.

24. Bui T, Kocher J, Li Y, et al. Median infectious dose of human norovirus Gll.4 in gnotobiotic pigs is decreased by simvastatin treatment and increased by age. J Gen Virol. 2013;94(Pt 9):20052016. doi:10.1099/vir.0.054080-0.

25. Souza M, Azevedo MS, Jung K, Cheetham S, Saif LJ. Pathogenesis and immune responses in gnotobiotic calves after infection with the genogroup II.4-HS66 strain of human norovirus. J Virol. 2008;82(4):1777-1786. doi:10.1128/JVI.01347-07.

26. Hsu CC, Piotrowski SL, Meeker SM, Smith KD, MaggioPrice L, Treuting PM. Histologic lesions induced by murine norovirus infection in laboratory mice. Vet Pathol. 2016. doi:10.1177/0300985815618439.

27. Zonta W, Mauroy A, Farnir F, Thiry E. Comparative virucidal efficacy of seven disinfectants against murine norovirus and feline calicivirus, surrogates of human norovirus. Food Environ Virol. 2016;8(1):1-12. doi:10.1007/s12560-015-9216-2.

28. Arthur SE, Gibson KE. Environmental persistence of Tulane virus - a surrogate for human norovirus. Can J Microbiol. 2016;62(5):449454. doi:10.1139/cjm-2015-0756.

29. Richards GP. Critical review of norovirus surrogates in food safety research: rationale for considering volunteer studies. Food Environ Virol. 2012;4(1):6-13. doi:10.1007/s12560-011-9072-7.

30. Kapikian AZ, Wyatt RG, Dolin R, Thornhill TS, Kalica AR, 
Chanock RM. Visualization by immune electron microscopy of a 27-nm particle associated with acute infectious nonbacterial gastroenteritis. J Virol. 1972;10(5):1075-1081.

31. Division of Viral Diseases NCfl, Respiratory Diseases CfDC, Prevention. Updated norovirus outbreak management and disease prevention guidelines. MMWR Recomm Rep. 2011;60(RR-3):118.

32. Hall AJ, Lopman BA, Payne DC, et al. Norovirus disease in the United States. Emerg Infect Dis. 2013;19(8):1198-1205. doi:10.3201/eid1908.130465.

33. Ajami NJ, Kavanagh OV, Ramani S, et al. Seroepidemiology of norovirus-associated travelers' diarrhea. J Travel Med. 2014;21(1):6-11. doi:10.1111/jtm.12092.

34. Lindesmith LC, Beltramello M, Donaldson EF, et al. Immunogenetic mechanisms driving norovirus Gll.4 antigenic variation. PLoS Pathog. 2012;8(5):e1002705. doi:10.1371/journal.ppat.1002705.

35. Fu JG, Ai J, Qi X, Zhang J, Tang FY, Zhu YF. Emergence of two novel norovirus genotype II.4 variants associated with viral gastroenteritis in China. J Med Virol. 2014;86(7):1226-1234. doi:10.1002/jmv.23799.

36. Green KY. Norovirus infection in immunocompromised hosts. Clin Microbiol Infect. 2014;20(8):717-723. doi:10.1111/14690691.12761

37. Cummins M, Ready D. Role of the Hospital Environment in Norovirus Containment. J Infect Dis. 2016;213 Suppl 1:S12-14. doi:10.1093/infdis/jiv529.

38. Wikswo ME, Cortes J, Hall AJ, et al. Disease transmission and passenger behaviors during a high morbidity Norovirus outbreak on a cruise ship, January 2009. Clin Infect Dis. 2011;52(9):11161122. doi:10.1093/cid/cir144.

39. Vega E, Barclay L, Gregoricus N, Shirley SH, Lee D, Vinje J. Genotypic and epidemiologic trends of norovirus outbreaks in the United States, 2009 to 2013. J Clin Microbiol. 2014;52(1):147155. doi:10.1128/JCM.02680-13.

40. Leshem E, Gastanaduy PA, Trivedi T, et al. Norovirus in a United States virgin islands resort: outbreak investigation, response, and costs. J Travel Med. 2016;23(5). doi:10.1093/jtm/taw040.

41. Widdowson MA, Glass R, Monroe $S$, et al. Probable transmission of norovirus on an airplane. JAMA. 2005;293(15):1859-1860. doi:10.1001/jama.293.15.1859.

42. Marks PJ, Vipond IB, Regan FM, Wedgwood K, Fey RE, Caul EO. A school outbreak of Norwalk-like virus: evidence for airborne transmission. Epidemiol Infect. 2003;131(1):727-736.

43. Atmar RL, Opekun AR, Gilger MA, et al. Determination of the $50 \%$ human infectious dose for Norwalk virus. J Infect Dis. 2014;209(7):1016-1022. doi:10.1093/infdis/jit620.

44. Roth AN, Karst SM. Norovirus mechanisms of immune antagonism. Curr Opin Virol. 2016;16:24-30. doi:10.1016/j. coviro.2015.11.005.

45. Lee RM, Lessler J, Lee RA, et al. Incubation periods of viral gastroenteritis: a systematic review. BMC Infect Dis. 2013;13:446. doi:10.1186/1471-2334-13-446.

46. Teunis PF, Sukhrie FH, Vennema H, Bogerman J, Beersma MF, Koopmans MP. Shedding of norovirus in symptomatic and asymptomatic infections. Epidemiol Infect. 2015;143(8):17101717. doi:10.1017/S095026881400274X.

47. Donaldson EF, Lindesmith LC, Lobue AD, Baric RS. Norovirus pathogenesis: mechanisms of persistence and immune evasion in human populations. Immunol Rev. 2008;225:190-211.

48. Eden JS, Hewitt J, Lim KL, et al. The emergence and evolution of the novel epidemic norovirus Gll.4 variant Sydney 2012. Virology. 2014;450-451:106-113. doi:10.1016/j.virol.2013.12.005.

49. Debbink K, Donaldson EF, Lindesmith LC, Baric RS. Genetic mapping of a highly variable norovirus Gll.4 blockade epitope: potential role in escape from human herd immunity. J Virol. 2012;86(2):1214-1226. doi:10.1128/JVI.06189-11.

50. Novella IS, Presloid JB, Taylor RT. RNA replication errors and the evolution of virus pathogenicity and virulence. Curr Opin Virol. 2014;9:143-147. doi:10.1016/j.coviro.2014.09.017.

51. Bull RA, White PA. Mechanisms of Gll.4 norovirus evolution. Trends Microbiol.2011;19(5):233-240. doi:10.1016/j.tim.2011.01.002.

52. Copper PD, Steiner-Pryor A, Scotti PD, Delong D. On the nature of poliovirus genetic recombinants. J Gen Virol. 1974;23(1):41-49. doi:10.1099/0022-1317-23-1-41.

53. Fu JG, Ai J, Zhang J, et al. Molecular epidemiology of genogroup II norovirus infection among hospitalized children with acute gastroenteritis in Suzhou (Jiangsu, China) from 2010 to 2013. J Med Virol. 2016;88(6):954-960. doi:10.1002/jmv.24429.

54. Bull RA, Tanaka MM, White PA. Norovirus recombination. J Gen Virol. 2007;88(Pt 12):3347-3359. doi:10.1099/vir.0.83321-0.

55. Eden JS, Tanaka MM, Boni MF, Rawlinson WD, White PA. Recombination within the pandemic norovirus Gll.4 lineage. J Virol. 2013;87(11):6270-6282. doi:10.1128/JVI.03464-12.

56. Puustinen L, Blazevic V, Huhti L, et al. Norovirus genotypes in endemic acute gastroenteritis of infants and children in Finland between 1994 and 2007. Epidemiol Infect. 2012;140(2):268-275. doi:10.1017/S0950268811000549.

57. Kaplan JE, Feldman R, Campbell DS, Lookabaugh C, Gary GW. The frequency of a Norwalk-like pattern of illness in outbreaks of acute gastroenteritis. Am J Public Health. 1982;72(12):1329-1332.

58. Turcios RM, Widdowson MA, Sulka AC, Mead PS, Glass RI. Reevaluation of epidemiological criteria for identifying outbreaks of acute gastroenteritis due to norovirus: United States, 19982000. Clin Infect Dis. 2006;42(7):964-969. doi:10.1086/500940.

59. Oshiro LS, Haley CE, Roberto RR, et al. A 27-nm virus isolated during an outbreak of acute infectious nonbacterial gastroenteritis in a convalescent hospital: a possible new serotype. J Infect Dis. 1981;143(6):791-795.

60. Vyas K, Atkinson C, Clark DA, Irish D. Comparison of five commercially available immunochromatographic tests for the detection of norovirus in faecal specimens. J Hosp Infect. 2015;91(2):176-178. doi:10.1016/j.jhin.2015.06.013.

61. Thery L, Bidalot M, Pothier P, Ambert-Balay K. Evaluation of immunochromatographic tests for the rapid detection of the emerging Gll.17 norovirus in stool samples, January 2016. Euro Surveill. 2016;21(4). doi:10.2807/1560-7917. ES.2016.21.4.30115.

62. Xia $M$, Farkas $T$, Jiang $X$. Norovirus capsid protein expressed in yeast forms virus-like particles and stimulates systemic and mucosal immunity in mice following an oral administration of raw yeast extracts. J Med Virol. 2007;79(1):74-83. doi:10.1002/ jmv.20762.

63. Jiang X, Wang M, Graham DY, Estes MK. Expression, self-assembly, and antigenicity of the Norwalk virus capsid protein. J Virol. 1992;66(11):6527-6532.

64. Lai H, Chen Q. Bioprocessing of plant-derived virus-like particles of Norwalk virus capsid protein under current Good Manufacture Practice regulations. Plant Cell Rep. 2012;31(3):573-584. doi:10.1007/s00299-011-1196-6.

65. Santi L, Batchelor L, Huang Z, et al. An efficient plant viral expression system generating orally immunogenic Norwalk viruslike particles. Vaccine. 2008;26(15):1846-1854. doi:10.1016/j. vaccine.2008.01.053.

66. Tan $M$, Jiang $X$. The $p$ domain of norovirus capsid protein forms a subviral particle that binds to histo-blood group antigen receptors. J Virol. 2005;79(22):14017-14030. doi:10.1128/JVI.79.22.1401714030.2005 .

67. Atmar RL, Bernstein DI, Harro CD, et al. Norovirus vaccine against experimental human Norwalk Virus illness. N Engl J Med. 2011;365(23):2178-2187. doi:10.1056/NEJMoa1101245.

68. LoBue AD, Lindesmith L, Yount B, et al. Multivalent norovirus vaccines induce strong mucosal and systemic blocking antibodies against multiple strains. Vaccine. 2006;24(24):5220-5234. doi:10.1016/j.vaccine.2006.03.080.

69. El-Kamary SS, Pasetti MF, Mendelman PM, et al. Adjuvanted intranasal Norwalk virus-like particle vaccine elicits antibodies and antibody-secreting cells that express homing receptors for mucosal and peripheral lymphoid tissues. J Infect Dis. 2010;202(11):16491658. doi:10.1086/657087.

70. Tacket CO, Sztein MB, Losonsky GA, Wasserman SS, Estes MK. Humoral, mucosal, and cellular immune responses to oral Norwalk virus-like particles in volunteers. Clin Immunol. 2003;108(3):241247. 